BRIEF COMMUNICATION

\title{
Novel metagenome-assembled genomes involved in the nitrogen cycle from a Pacific oxygen minimum zone
}

\author{
Xin Sun (iD ${ }^{1,2,3,4 \times}$ and Bess B. Ward (iD ${ }^{1}$
}

(c) The Author(s) 2021

Oxygen minimum zones (OMZs) are unique marine regions where broad redox gradients stimulate biogeochemical cycles. Despite the important and unique role of OMZ microbes in these cycles, they are less characterized than microbes from the oxic ocean. Here we recovered 39 high- and medium-quality metagenome-assembled genomes (MAGs) from the Eastern Tropical South Pacific OMZ. More than half of these MAGs were not represented at the species level among 2631 MAGs from global marine datasets. OMZ MAGs were dominated by denitrifiers catalyzing nitrogen loss and especially MAGs with partial denitrification metabolism. A novel bacterial genome with nitrate-reducing potential could only be assigned to the phylum level. A Marine-Group II archaeon was found to be a versatile denitrifier, with the potential capability to respire multiple nitrogen compounds including $\mathrm{N}_{2} \mathrm{O}$. The newly discovered denitrifying MAGs will improve our understanding of microbial adaptation strategies and the evolution of denitrification in the tree of life.

ISME Communications (2021)1:26; https://doi.org/10.1038/s43705-021-00030-2

\section{MAIN}

Oxygen minimum zones (OMZs) are unique oceanic regions with strong redox gradients. Anoxic zones in OMZs are hotspots for fixed nitrogen loss and production of the greenhouse gas $\mathrm{N}_{2} \mathrm{O}[1,2]$. Microbes in OMZs make important contributions to biogeochemistry, which motivates us to reconstruct metagenome-assembled genomes (MAGs) from the Eastern Tropical South Pacific (ETSP) OMZ (Fig. 1a, b). Among 147 recovered MAGs, we present 39 high- and medium-quality MAGs with completeness $>50 \%$ and contamination $<10 \%$ [3], including 8 archaeal and 31 bacterial MAGs (Fig. S1 and Table S1) representing 11 phyla (Fig. 1c). Methods of MAG construction and analysis are available in the supplement. We compared these new OMZ MAGs to 2631 MAGs recovered from the most comprehensive marine microbial metagenomic datasets (Tara Oceans) [4], which included OMZ and non-OMZ sites. More than half of these ETSP OMZ MAGs were not represented at the species level in the Tara Oceans dataset (Fig. 2 and Supplementary Methods). These 39 ETSP MAGs only represented up to $24 \%$ of the total microbial population (Fig. 1c), thus many more novel species in OMZs remain to be discovered. Seventeen ETSP OMZ MAGs were identified as the same species in regions, where $O M Z$ sites were included in the Tara Oceans dataset (Fig. 2), indicating adaptation to the unique $\mathrm{OMZ}$ environment and the necessity to explore these OMZ MAGs for novel taxa and functional potentials.

Taxonomy-resolved MAGs recovered here will allow linking previously measured biogeochemical cycling rates on the same cruise [5-10] to their microbial drivers. The most abundant fixed nitrogen in the ocean, nitrate, is produced via nitrification. The first step of nitrification, ammonia oxidation, is mainly performed by marine ammonia-oxidizing archaea (AOA) [11], and then nitrite-oxidizing bacteria (NOB) oxidize nitrite into nitrate. Novel niches of NOB were discovered by analyzing the two NOB MAGs from this dataset [12]. Kinetics experiments at other $\mathrm{OMZ}$ stations suggested distinct oxygen affinities of $A O A$ and NOB [13-15]. In anoxic waters, ammonia oxidation rates were undetectable, but nitrite oxidation rates were high $\left(>100 \mathrm{nM} \mathrm{d}^{-1}\right)$ at the same station [6], where MAGs were recovered. Consistently, Thaumarchaeota MAGs (AOAs) were nearly absent (only $A O A-2$ had a relative abundance higher than $0.01 \%$ ) and NOB MAGs (NOB-1 and NOB-2) were much more abundant than $A O A$ in the anoxic core (Fig. 1d). MAGs in this study will provide opportunities to discover novel processes and adaptation strategies.

Most MAGs had their highest relative abundances in the anoxic zone (Fig. 1c). Many of them contribute to the loss of fixed nitrogen, which occurs by denitrification (the sequential reduction of nitrate to nitrite, $\mathrm{NO}, \mathrm{N}_{2} \mathrm{O}$, and finally $\mathrm{N}_{2}$ ) and anammox (anaerobic oxidation of ammonium to $\mathrm{N}_{2}$ ). Measured nitrate reduction rates at this $[5,8]$ and other $[16,17]$ nearby stations were much larger than rates of any subsequent denitrification steps (e.g., nitrite reduction to $\mathrm{N}_{2} \mathrm{O}$ or to $\mathrm{N}_{2}$ ). Consistently, preliminary prediction of metabolisms shows that more than half of the MAGs found here contained nar, which encodes nitrate reduction, and one-third of those contained only nar and none of the other denitrification genes (i.e., they are nitrate-reducing specialists) (Fig. 2). Consistently, a previous study found that nar dramatically outnumbered the other

\footnotetext{
${ }^{1}$ Department of Geosciences, Guyot Hall, Princeton University, Princeton, NJ, USA. ²Present address: Department of Ecology and Evolutionary Biology, Yale University, New Haven,

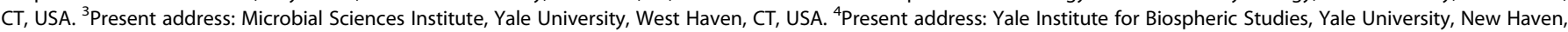
CT, USA. ${ }^{凶}$ email: x.sun@yale.edu
} 


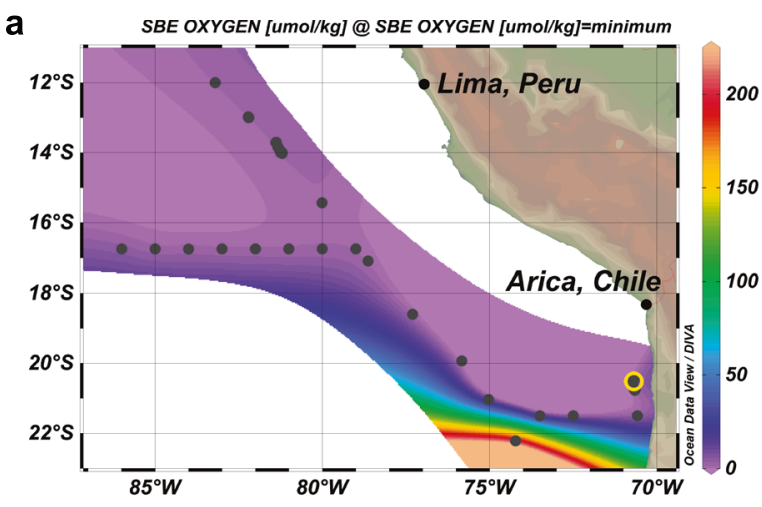

C

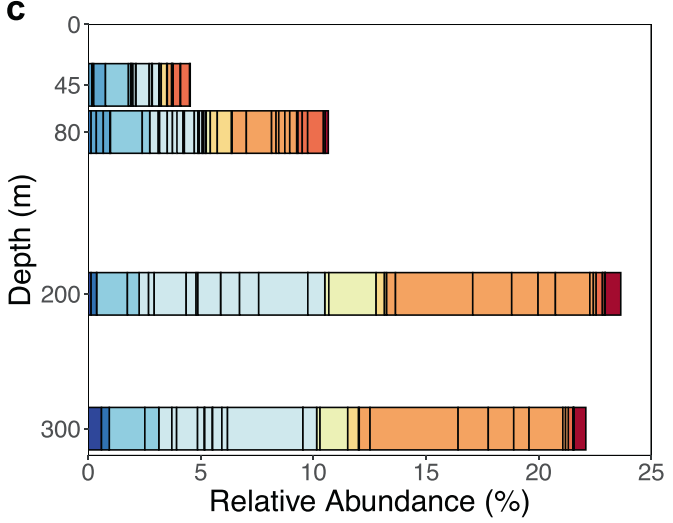

e

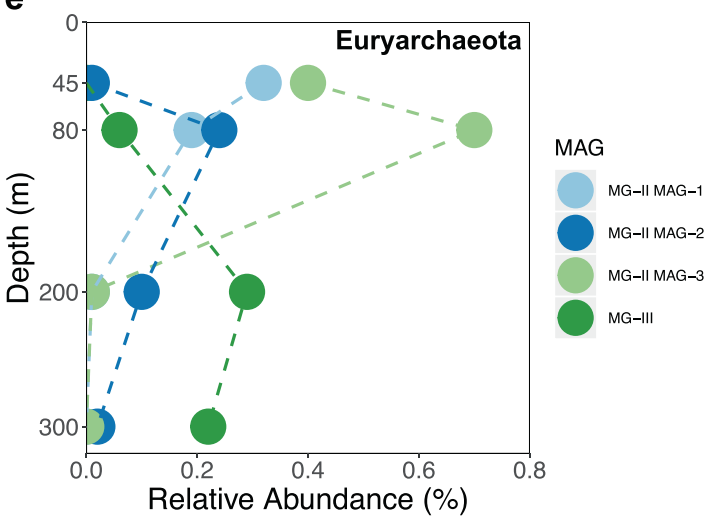

b
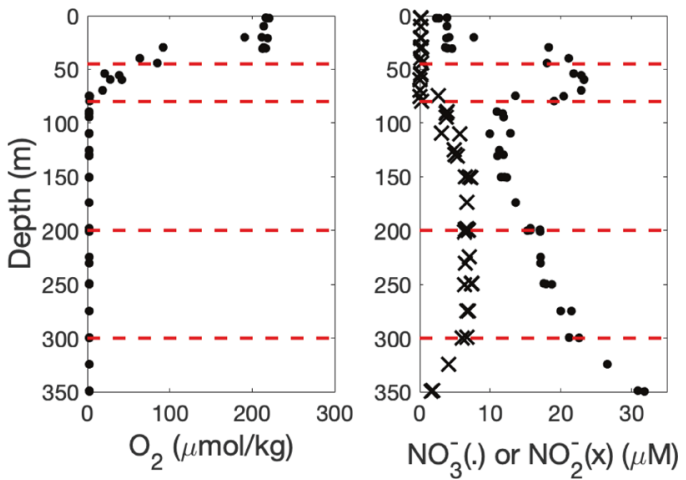

d

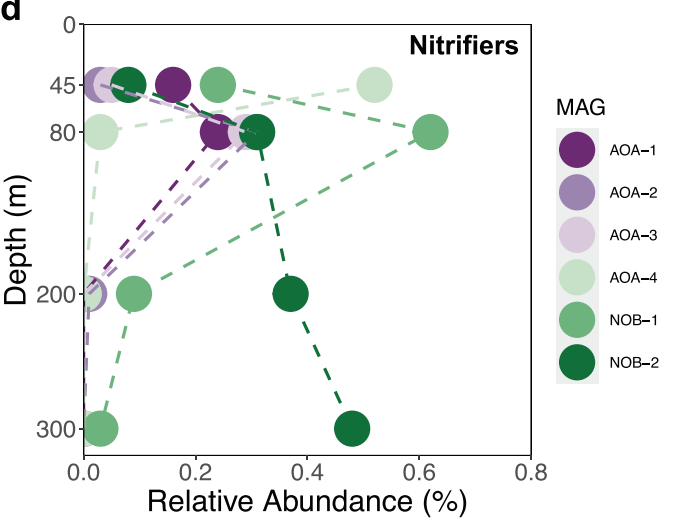

f

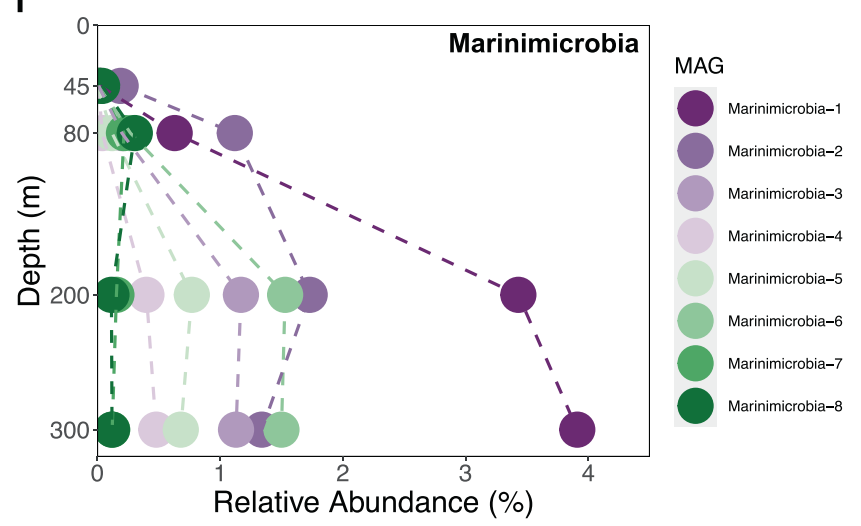

Fig. 1 Sampling station information and relative abundances of MAGs. a Sampling station (yellow circle) in the ETSP OMZ and the minimum oxygen concentration at the time of the cruise. b Depth profiles of oxygen, nitrate, and nitrite reported previously [7] at the sampling station and the four sample depths indicated by red dashed lines $(45,80,200$, and 300 m). c Relative abundances of MAGs in the ETSP OMZ grouped by phylum. $\mathbf{d}-\mathbf{f}$ The distribution of individual MAGs in four phyla discussed in the text. The relative abundance of NOB-1 and NOB-2 was reported previously [12].

denitrification genes in contigs from the Eastern Tropical North Pacific (ETNP) OMZ [18]. Indeed, four of the five most abundant MAGs in the anoxic core were nitrate-reducing specialists (Fig. 2). The fifth was an anammox MAG, which was only assigned to the genus level (Candidatus Scalindua) in GTDB and was not represented at the species level in the Tara Oceans dataset (Table S1). However, this anammox MAG was highly related to 20 anammox single-cell amplified genomes (SAGs) from the ETNP OMZ [19]. The anammox MAG had at least $90 \%$ average nucleotide identity (ANI) to the SAGs, with the highest ANI $(98.8 \%)$ to SAG K21. Consistent with the previous work [19], the anammox MAG also encoded cyanase, indicating its potential of using organic nitrogen substrates. The most abundant nitrate reducer MAG here is Marinimicrobia-1 (Fig. 1), which belongs to the newly proposed phylum Candidatus Marinimicrobia [20]. Notably, one nitrate reducer can only be assigned to phylum level (Candidatus Wallbacteria) and was not present in the Tara Oceans MAGs (Table S1).

We also identified a novel archaeal MAG possessing multiple denitrification genes. MG-II MAG-2 encoded Nar alpha and beta subunits, nitrate/nitrite transporters, copper-containing nitrite reductase, and $\mathrm{N}_{2} \mathrm{O}$ reductase (Fig. 2). Two MAGs from the Tara Oceans metagenomes (Table S1) were identified as the same species as MGII MAG-2. TOBG_NP-110 (ANI to MG-II MAG-2=99.8\%) from the North Pacific encoded Nar and nitrate/nitrite transporters, and TOBG_SP-208 (ANI to MG-II MAG-2=99.6\%) from the South Pacific also contained the same denitrification genes as MG-II MAG-2 (Table S2). In addition, two MG-II SAGs (AD-615-F09 and AD-613O09) were found at a different station of the ETSP OMZ sampled on the same cruise as this study [21]. Partial 16S rRNA genes of both SAGs are $100 \%$ identical to that of MG-II MAG-2 (alignment length $=200 \mathrm{bp}$ for AD-615-F09 and $183 \mathrm{bp}$ for AD-613-O09), but only AD- 


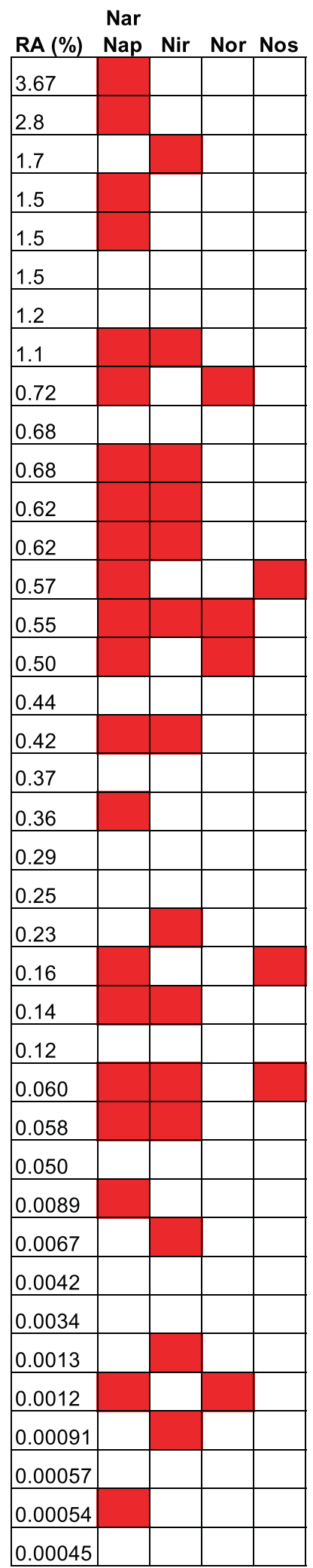

Marinimicrobia-1

Gammaproteobacteria-3

Anammox

Marinimicrobia-2

Marinimicrobia-6

SAR324 MAG-1

Alphaproteobacteria-2

Marinimicrobia-3

Marinimicrobia-5

Pelagibacter-3

Thioglobaceae-1

Thioglobaceae-3

Actinobacteriota

SAR324 MAG-2

Thioglobaceae-4

Gammaproteobacteria-1

Marinimicrobia-4

NOB-2

Pelagibacter-1

Wallbacteria

Verrucomicrobiota

MG-III

Alphaproteobacteria-1

Planctomycetota

Marinimicrobia-7

Marinimicrobia-8

MG-II MAG-2

NOB-1

Pelagibacter-2

Thioglobaceae-2

AOA-2

MG-II MAG-3

MG-II MAG-1

AOA-1

Gammaproteobacteria-2

AOA-3

Chloroflexota

Thioglobaceae-5

AOA-4

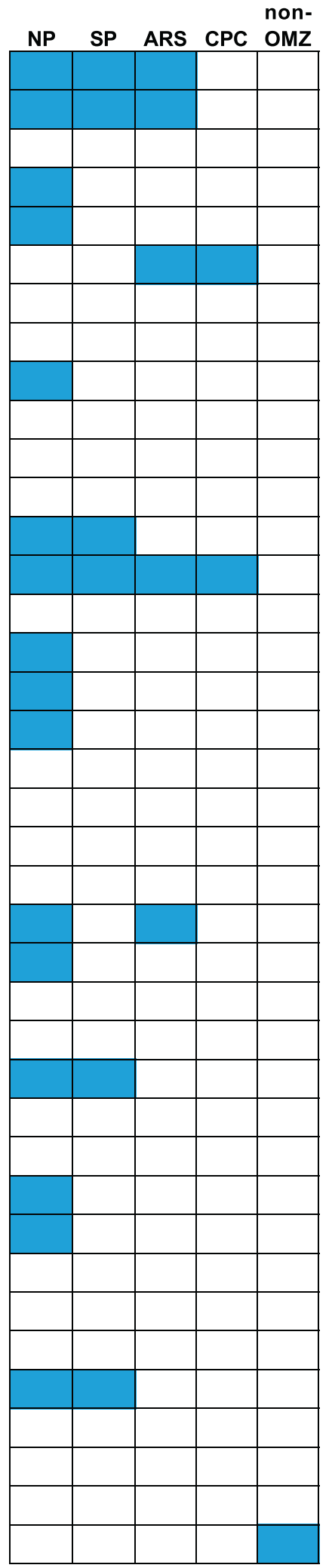

Fig. 2 Preliminary prediction of nitrogen cycling metabolisms and representation of MAGs in global ocean. Presence (red) and absence (white) of genes involved in the denitrification pathway (Nar or Nap nitrate reductase, Nir nitrite reductase, Nor nitric oxide reductase, Nos $\mathrm{N}_{2} \mathrm{O}$ reductase) for each MAG. Relative abundance (RA) of MAGs in the anoxic core of the ETSP OMZ (averaged between 200 and $300 \mathrm{~m}$ ). MAGs from this study are ordered by their relative abundance in the anoxic core. Presence (blue) and absence (white) of MAGs from Tara Oceans datasets, which were identified as the same species with MAGs from this study. Tara Oceans regions, where Tara Oceans MAGs were originally found, include four regions with OMZ sites: NP North Pacific region, SP South Pacific region, ARS Arabian Sea region, CPC Chile-Peru Coastal region, and non-OMZ regions including North Atlantic, South Atlantic, East Africa Coastal, Mediterranean, Red Sea, and Indian Ocean regions. 
615-F09 might be the same species as MG-II MAG-2 based on ANI analyses (MG-II MAG-2 had 99.5\% ANI to AD-615-F09, and $80.9 \%$ to AD-613-009). Both SAGs also encoded $\mathrm{Nar}$ and nitrate/nitrite transporters [21]. The absence of other denitrification genes may be due to the low completeness of the two SAGs (completeness = $5.61 \%$ for both SAGs) [21]. Nitrite reductase and $\mathrm{N}_{2} \mathrm{O}$ reductase genes were located on the same contig in both MG-II MAG-2 and TOBG_SP-208 (Table S2). MG-II MAG-2 and TOBG_SP-208 had low contamination ( $1.9 \%$ and $0.8 \%$, respectively), and their contigs with nitrite reductase and $\mathrm{N}_{2} \mathrm{O}$ reductase genes contained single-copy marker genes present only once in each MAG (Supplementary Methods). Although these results suggest a nearly complete denitrification metabolism in MG-II archaea, especially $\mathrm{N}_{2} \mathrm{O}$ consumption metabolism, methods besides metagenomics (e.g. reconstructing SAGs with high completeness) are highly recommended to rule out possible artifacts introduced by metagenomic binning and confirm the presence of these genes and their denitrification activity. Nonetheless, MG-II MAG-2 was present (Fig. 1e) and transcriptionally active in both Pacific OMZs (Fig. S2), indicating its adaptation to low oxygen environments. The MG-III MAG did not have any denitrification genes but was abundant in the anoxic zone (Figs. 1e and 2). It had a GC value (43.2\%) distinct from all other known MG-III MAGs [22] and is the most complete (86.0\%) and the least contaminated (0\%) (Table S1) among all reported MG-III MAGs, indicating that MG-III is a novel archaeon in this group. Bacterial and archaeal MAGs recovered here implied that nitrogen metabolisms were present in more microbial lineages than previously thought. Further analyses of these MAGs will shed light on adaptation strategies in the unique $\mathrm{OMZ}$ environment and novel functions related to important element cycles.

\section{DATA AVAILABILITY}

Raw metagenomic reads used to construct MAGs in this paper were submitted to NCBI with the accession numbers SRR14610252, SRR14610253, SRR14610254, and SRR14610255. MAGs analyzed in this paper were deposited at https://figshare.com/ articles/MAGs_from_ETSP_OMZ/12291281.

\section{REFERENCES}

1. Codispoti LA, Brandes JA, Christensen JP, Devol AH, Naqvi SWA, Paerl HW, et al. The oceanic fixed nitrogen and nitrous oxide budgets: moving targets as we enter the anthropocene? Sci Mar. 2001;65:85-105.

2. Ji Q, Buitenhuis E, Suntharalingam P, Sarmiento JL, Ward BB. Global nitrous oxide production determined by oxygen sensitivity of nitrification and denitrification. Global Biogeochem Cycles. 2018;32:1790-802.

3. Bowers RM, Kyrpides NC, Stepanauskas R, Harmon-Smith M, Doud D, Reddy T, et al. Minimum information about a single amplified genome (MISAG) and a metagenome-assembled genome (MIMAG) of bacteria and archaea. Nat Biotechnol. 2017;35:725-31.

4. Tully BJ, Graham ED, Heidelberg JF. The reconstruction of 2,631 draft metagenomeassembled genomes from the global oceans. Sci Data. 2018;5:170203.

5. Ji Q, Babbin A, Jayakumar A, Oleynik S, Ward BB. Nitrous oxide production by nitrification and denitrification in the Eastern Tropical South Pacific oxygen minimum zone. Geophys Res Lett. 2015;42:755-64.

6. Peng X, Fuchsman CA, Jayakumar A, Warner MJ, Devol AH, Ward BB. Revisiting nitrification in the Eastern Tropical South Pacific: a focus on controls. J Geophys Res Ocean. 2016;121:1667-84.

7. Sun X, Jayakumar A, Ward BB. Community composition of nitrous oxide consuming bacteria in the oxygen minimum zone of the Eastern Tropical South Pacific. Front Microbiol. 2017;8:1-11.

8. Babbin AR, Peters BD, Mordy CW, Widner B, Casciotti KL, Ward BB. Multiple metabolisms constrain the anaerobic nitrite budget in the Eastern Tropical South Pacific. Global Biogeochem Cycles. 2017;31:258-71.

9. Casciotti KL, Forbes M, Vedamati J, Peters BD, Martin TS, Mordy CW. Nitrous oxide cycling in the Eastern Tropical South Pacific as inferred from isotopic and isotopomeric data. Deep Res Part II Top Stud Oceanogr. 2018;156:155-67.

10. Widner B, Mordy CW, Mulholland MR. Cyanate distribution and uptake above and within the Eastern Tropical South Pacific oxygen deficient zone. Limnol Oceanogr. 2018;63:S177-92.
11. Martens-Habbena W, Berube PM, Urakawa H, de la Torre JR, Stahl DA. Ammonia oxidation kinetics determine niche separation of nitrifying Archaea and Bacteria. Nature. 2009;461:976-9.

12. Sun X, Kop L, Lau M, Frank J, Jayakumar A, Lücker S, et al. Uncultured Nitrospinalike species are major nitrite oxidizing bacteria in oxygen minimum zones. ISME J. 2019;13:2391-402.

13. Bristow LA, Dalsgaard T, Tiano L, Mills DB, Bertagnolli AD, Wright JJ, et al. Ammonium and nitrite oxidation at nanomolar oxygen concentrations in oxygen minimum zone waters. Proc Natl Acad Sci. 2016;113:10601-6.

14. Sun X, Ji Q, Jayakumar A, Ward BB. Dependence of nitrite oxidation on nitrite and oxygen in low oxygen seawater. Geophys Res Lett. 2017;44:7883-91.

15. Sun X, Frey C, Garcia-Robledo E, Jayakumar A, Ward BB. Microbial niche differentiation explains nitrite oxidation in marine oxygen minimum zones. ISME J. 2021;15:1317-29.

16. Ward BB, Tuit CB, Jayakumar A, Rich JJ, Moffett J, Naqvi SWA. Organic carbon, and not copper, controls denitrification in oxygen minimum zones of the ocean. Deep Sea Res Part I Oceanogr Res Pap. 2008;55:1672-83.

17. Lipschultz F, Wofsy SC, Ward BB, Codispoti LA, Friedrich G, Elkins JW. Bacterial transformations of inorganic nitrogen in the oxygen-deficient waters of the Eastern Tropical South Pacific Ocean. Deep Sea Res Part A, Oceanogr Res Pap. 1990;37:1513-41.

18. Fuchsman CA, Devol AH, Saunders JK, Mckay C, Rocap G. Niche partitioning of the $\mathrm{N}$ cycling microbial community of an offshore oxygen deficient zone. Front Mar Sci. 2017;8:1-18.

19. Ganesh S, Bertagnolli AD, Bristow LA, Padilla CC, Blackwood N, Aldunate M, et al. Single cell genomic and transcriptomic evidence for the use of alternative nitrogen substrates by anammox bacteria. ISME J. 2018;12:2706-22.

20. Hawley AK, Nobu MK, Wright JJ, Durno WE, Morgan-Lang C, Sage B, et al. Diverse Marinimicrobia bacteria may mediate coupled biogeochemical cycles along ecothermodynamic gradients. Nat Commun. 2017;8:1-9.

21. Plominsky AM, Trefault N, Podell S, Blanton JM, De la Iglesia R, Allen EE, et al. Metabolic potential and in situ transcriptomic profiles of previously uncharacterized key microbial groups involved in coupled carbon, nitrogen and sulfur cycling in anoxic marine zones. Environ Microbiol. 2018;20:2727-42.

22. Haro-Moreno JM, Rodriguez-Valera F, López-García P, Moreira D, Martin-Cuadrado A-BB. New insights into marine group III Euryarchaeota, from dark to light. ISME J. 2017;11:1102-17.

\section{ACKNOWLEDGEMENTS}

We would like to acknowledge all scientists and the crew of the R/V Nathaniel B. Palmer for assistance, especially Amal Jayakumar for collecting DNA samples. We thank Jeroen Frank and Sebastian Lücker for providing metagenomic pipeline in the first study from this dataset, and Wei Wang for his help in the Princeton Genomics Core Facility. This work was funded by NSF Grant OCE-1029951 to BBW. XS was partially supported by the High Meadows Environmental Institute at Princeton University through the Mary and Randall Hack '69 Research Fund.

\section{AUTHOR CONTRIBUTIONS}

XS and BBW conceptualized the study. XS and BBW performed research. XS analyzed the data. XS and BBW wrote the paper.

\section{COMPETING INTERESTS}

The authors declare no competing interests.

\section{ADDITIONAL INFORMATION}

Supplementary information The online version contains supplementary material available at https://doi.org/10.1038/s43705-021-00030-2.

Correspondence and requests for materials should be addressed to X.S.

Reprints and permission information is available at http://www.nature.com/ reprints

Publisher's note Springer Nature remains neutral with regard to jurisdictional claims in published maps and institutional affiliations. 
Open Access This article is licensed under a Creative Commons At c) Atribution 4.0 International License, which permits use, sharing,
adaptation, distribution and reproduction in any medium or format, as long as you give appropriate credit to the original author(s) and the source, provide a link to the Creative Commons license, and indicate if changes were made. The images or other third party material in this article are included in the article's Creative Commons license, unless indicated otherwise in a credit line to the material. If material is not included in the article's Creative Commons license and your intended use is not permitted by statutory regulation or exceeds the permitted use, you will need to obtain permission directly from the copyright holder. To view a copy of this license, visit http://creativecommons. org/licenses/by/4.0/.

(c) The Author(s) 2021 